International Journal of Engineering, Science And Technology Vol. 3, No. 1, 2011, pp. 52-68
INTERNATIONAL

JOURNAL OF

ENGINEERING,

SCIENCE AND

TECHNOLOGY

www.ijest-ng.com

(C) 2011 MultiCraft Limited. All rights reserved

\title{
Analysis of particles loaded fiber composites for the evaluation of effective material properties with the variation of shape and size
}

\author{
V. K. Srivastava ${ }^{1}$, U. Gabbert ${ }^{2}$, H. Berger ${ }^{2}$, Shraddha $\operatorname{Singh}^{3}$

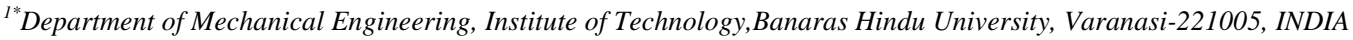 \\ ${ }^{2}$ Institute of Mechanics, Otto- von Guericke University of Magdeburg, Universitaetsplatz 2, D-39106, Magdeburg, GERMANY \\ ${ }^{3}$ School of Materials Science \& Technology, Institute of Technology, Banaras Hindu University, Varanasi-221005, INDIA \\ *Corresponding author: e-mail: vijayks210@ gmail.com, Tel. +91-542-6702833, Fax.+91-542-2368428
}

\begin{abstract}
The effective material properties are predicted for composites with different shape and size of inclusions such as cylindrical fibers, spherical and elliptical particles and cylindrical fibers with hemispherical ends. The analysis is based on a numerical homogenization technique using finite element method in connection with three-dimensional representative volume element models. Investigations are carried out to study the influence of various parameters like volume fraction, aspect ratio and particle distribution. Results are discussed and compared.
\end{abstract}

Keywords: Finite element analysis; Fibers; Volume fraction; Mechanical properties

\section{Introduction}

The development and characterization of multifunctional composite materials have been focus by the research community in recent years with emphasis on the potential applications and benefits for various industries. Thermo-elastic composites constitute an important class of materials with a wide variety of applications ranging from aerospace structures and electronic printed circuit boards to recreational and commercial equipment. Some of the most important and useful properties of these composites are lightweight, high strength and stiffness, excellent frictional properties, good resistance to fatigue and retention of these properties at high temperatures. The combination of these properties has placed thermo-elastic composites at first rank among materials used for heat shields, leading edges, re-entry tips, rocket nozzles and brakes for military and advanced civilian aircrafts. The effective thermo-mechanical properties of the composite depend upon properties of the constituents and the fiber volume fraction.

Many authors have developed techniques to study the elastic behavior of fibrous composites. They take into account the existence of an intermediate layer between the matrix and the fiber (Agbossou et al, 1997). These thin layers are called interphases or interfacial zones between fiber and matrix. The effective utilization of the fiber reinforced composites depends on efficient load transfer from the matrix to fibers through these interphases. These interphases are formed due to, for example, chemical reaction between the matrix and fiber materials or the use of protective coatings on the fiber during manufacturing. Although small in thickness, interphases can significantly affect the overall mechanical properties of the fiber-reinforced composites. It is the weakest link in the load path, and consequently most failures in fiber reinforced composites, such as debonding, fiber pullout, and matrix cracking, occur in or near this region. Thus, it is crucial to fully understand the mechanism and effects of the interphases on the overall material properties of fiber reinforced composites. Several homogenization techniques have been developed to obtain a suitable constitutive model to be inserted at the macroscopic level. Homogenization is a mechanics based modeling scheme that transforms a body of a heterogeneous material into a constitutively equivalent body of a homogeneous material, where the total energy stored in both systems is approximately same. The macroscopic properties are determined by a homogenization process, which yields the effective stresses and strains acting on the effective, homogenized sample of material. The sample of material is often called as statistically representative volume element (RVE) or unit cell (Berger et al, 2005). The RVE generation of homogenized composites and its representative is presented in Figs. $1 \& 2$. 


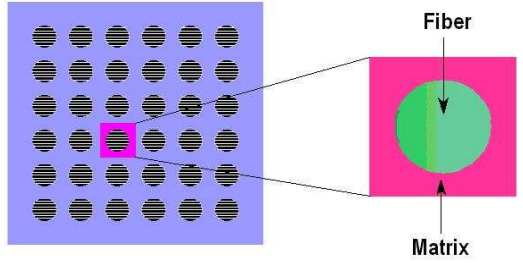

Fig. 1. Generation of RVE from a given composite

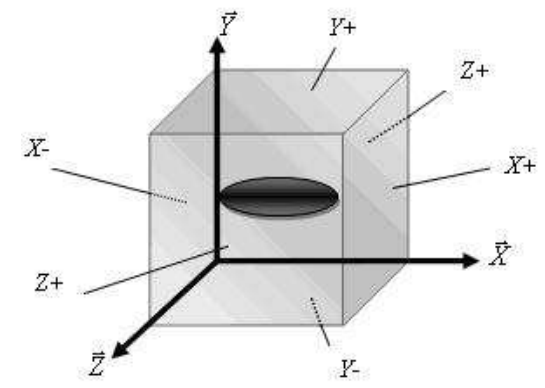

Fig. 2. Representation of surfaces of RVE

Modeling and prediction of the overall elastic-plastic response and local damage mechanisms in heterogeneous materials in general and particle-reinforced composites in particular, is a very complex problem. Analytical and empirical models provide an effective way for predicting the properties of composites from the known properties of its constituents, especially for simple configurations of the phases. Even if analytical and semi analytical models have been developed to homogenize fiber composites, they are often reduced to specific cases. A finite element analysis was attempted to describe the behavior of these materials, because there is no restriction on the geometry, size, material properties and number of phases in the composite (Berger et al, 2006). Kari et al, 2007, have studied the influence of randomly distributed spherical and cylindrical particle reinforced composites using numerical homogenization techniques with periodic boundary conditions, as shown in Fig. 3.

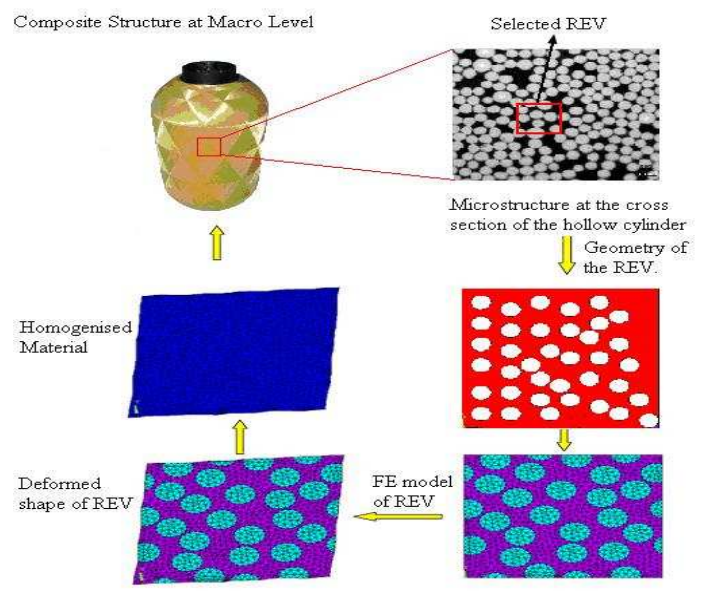

Fig. 3. Steps of numerical homogenization technique

In order to determine the best predictions of the elastic properties of an aligned unidirectional fiber reinforced composite, spatial distribution of the reinforcing fibers should be considered. The random nature of the transverse packing of the composite had a significant influence on the transverse elastic constants; and to predict them accurately, this microstructure aspect have to be considered. Several authors have reported the behavior of the composite materials with multi-scale approach. The overall properties of the composites that are obtained from the experimental results are well fitted with the investigations made either through the micro or macro mechanical analysis. The propagation of interfacial cracking or de-cohesion at fiber-matrix interfaces has been successfully modeled by a number of researchers using the cohesive volumetric finite element methods (Hashin, 2002, Kari 2006, Kari et al, 2007, Liu et al, 2000, Ramos et al, 2001, Wang 1998). A majority of these studies have been used for unit cell models, which assume that the material is constituted of periodic repetition of single cells. Due to lack of structural simplicity 
of continuous fiber composites or laminates, the finite element method (FEM) are often more suitable than analytical modeling for multiphase materials. Therefore, finite element method is used to determine the effective properties of the multi-shaped fiber composites.

In this paper, the effective material properties of randomly distributed particle loaded composites were calculated by considering a representative volume element (RVE) approach. Firstly, considered the existence of a RVE, and consequently adopted the deterministic, homogeneous continuum theories, which do not clearly account for random microstructures. In generating the RVE approach, consider; (i) statistical homogeneity representative and (ii) select a sufficiently large size of the RVE relative to the size of the inclusion to ensure the independence of the boundary conditions. Based on a unit cell model with appropriate periodic boundary conditions and load cases the finite element method was used to calculate the full set of effective coefficients of the composite. General numerical homogenization concept was used, which can be extended for the composites with arbitrary inclusions. The material properties for all analysis are listed in Table-1.

Table 1. Material parameters used for the analysis

\begin{tabular}{|c|c|c|}
\hline Material & Young's Modulus (GPa) & Poisson's Ratio \\
\hline Fiber/ Particle (SiC) & 450 & 0.17 \\
\hline Matrix (Aluminum Alloy) & 70 & 0.3 \\
\hline
\end{tabular}

\section{Constitutive equations and unit cell models}

The generalized Hooke's law can be formulated to correlate the stiffness matrix $\left(\mathrm{C}_{\mathrm{ij}}\right)$, average stress $\sigma_{\mathrm{ij}}$ and strain $\varepsilon_{\mathrm{ij}}$; for homogenized composites as (Berger et al, 2006)

$$
\left(\begin{array}{l}
\sigma_{1} \\
\sigma_{22} \\
\sigma_{33} \\
\sigma_{23} \\
\sigma_{31} \\
\sigma_{12}
\end{array}\right)=\left(\begin{array}{llllll}
\mathrm{C}_{11} & \mathrm{C}_{12} & \mathrm{C}_{13} & \mathrm{C}_{14} & \mathrm{C}_{15} & \mathrm{C}_{16} \\
& \mathrm{C}_{22} & \mathrm{C}_{23} & \mathrm{C}_{24} & \mathrm{C}_{25} & \mathrm{C}_{26} \\
& & \mathrm{C}_{33} & \mathrm{C}_{34} & \mathrm{C}_{35} & \mathrm{C}_{36} \\
& & & \mathrm{C}_{44} & \mathrm{C}_{45} & \mathrm{C}_{46} \\
& & & & \mathrm{C}_{55} & \mathrm{C}_{56} \\
& & & & & \mathrm{C}_{66}
\end{array}\right)\left(\begin{array}{c}
\varepsilon_{11} \\
\varepsilon_{22} \\
\varepsilon_{33} \\
\varepsilon_{23} \\
\varepsilon_{31} \\
\varepsilon_{12}
\end{array}\right)
$$

For the transversely isotropic material, the behavior of the composite is governed by five independent constants and all others are nullified because the transversely isotropic material is having one axis of revolution in addition three symmetry planes that are mutually orthogonal. The stiffness matrix remains unchanged due to an arbitrary rotation of the reference system about this axis. The use of this property leads to:

$$
\mathrm{C}_{11}=\mathrm{C}_{23}, \quad \mathrm{C}_{22}=\mathrm{C}_{11}, \quad \mathrm{C}_{44}=\mathrm{C}_{55} \quad \text { and C66 }=\left(\mathrm{C}_{11}-\mathrm{C}_{12}\right) / 2
$$

The relations between the effective elastic modulus and stiffness constants can be written as,

$$
\begin{aligned}
\mathrm{E}_{33} & =\mathrm{C}_{33}+\frac{2\left(C_{13}\right)^{2}}{C_{11}+C_{12}} \\
v_{13} & =\frac{C_{13}}{C_{11}+C_{13}} \\
\mathrm{E}_{11} & =\mathrm{C}_{11}+\frac{\left(C_{23}\right)^{2}\left(C_{11}-2 C_{12}\right)+C_{33}\left(C_{12}\right)^{2}}{\left(C_{23}\right)^{2}-C_{33} C_{11}} \\
v_{12} & =\frac{\left(C_{23}\right)^{2}-C_{33} C_{12}}{\left(C_{23}\right)^{2}-C_{33} C_{11}} \\
\mathrm{G}_{23} & =\mathrm{C}_{44}
\end{aligned}
$$




$$
\mathrm{G}_{12}=\frac{1}{2}\left(C_{11}-C_{12}\right)
$$

and,

$$
\mathrm{K}_{33}=\frac{1}{2}\left(C_{11}+C_{12}\right)
$$

where, $\mathrm{E}_{33}=$ Longitudinal Young's modulus, $\mathrm{E}_{11}=$ Transverse Young's modulus,

$v_{13}=$ Longitudinal Poisson's ratio, $v_{12}=$ Transverse Poisson's ratio,

$\mathrm{G}_{23}=$ Out-of-plane shear modulus or longitudinal shear modulus,

$\mathrm{G}_{12}=$ In-plane shear modulus or transverse shear modulus,

$\mathrm{K}_{33}=$ Bulk modulus

Finite RVE technique was used to define the material properties with the help of five independent elastic constants by considering randomly distributed different shaped reinforced with periodic boundary conditions. The appropriate boundary condition was applied to the unit cell in order to calculate effective coefficient. The effective coefficients such as $\mathrm{C}_{13}$ and $\mathrm{C}_{33}$ can be evaluated by averaged non-zero stress and strain vector.

\subsection{Periodic boundary conditions applied to RVE}

Three-dimensional composite materials can be represented as a periodic array of the RVEs. The periodic boundary conditions can be applied to the RVE models. It is assumed that, when the deformation caused by the load is experienced by each RVE in the composite, then there is no separation or overlap between the neighboring RVEs after deformation and the average mechanical properties of the RVE are equal to the average properties of the particular composite. Fig. 4 shows the RVEs containing different types of inclusions, which are placed suitably within the different positions. When the load is applied to the RVE, it is transferred to the reinforcing material through the inclusion-matrix interface. This is made possible by joining the node points of the inclusion with the node points of the matrix material so that transfer of load within the
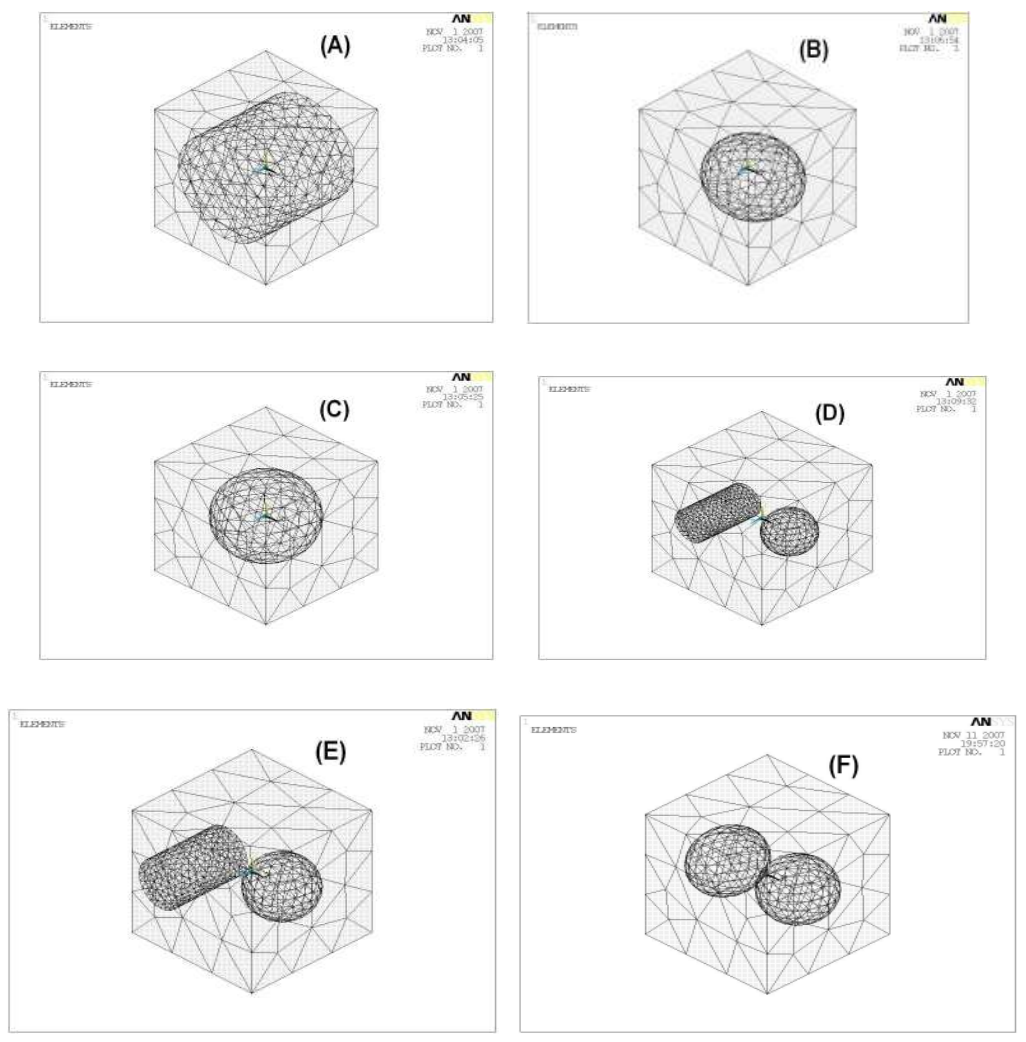

Fig. 4. Complete meshed RVE models containing different size, shape, volume fraction and distribution of particles within the matrix material. (a) Cylinder (b) Ellipse (c) Sphere (d) Cylinder and Sphere (e) Cylinder and Ellipse (f) Sphere and Ellipse 

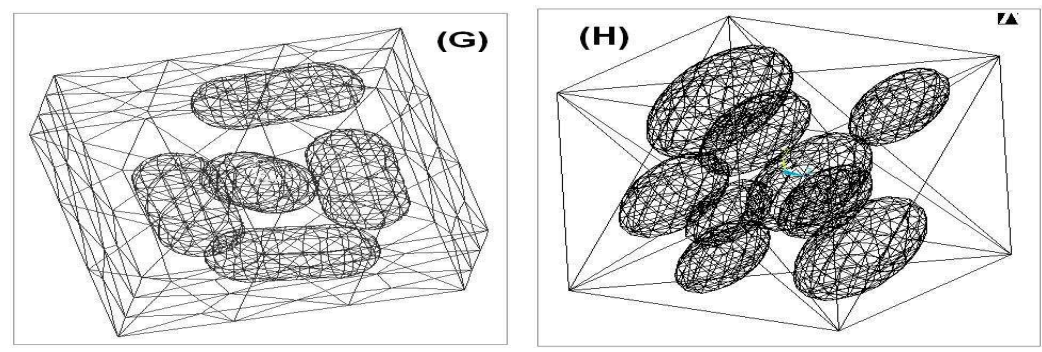

Fig. 4. Complete meshed RVE models containing different size, shape, volume fraction and distribution of particles within the matrix material. (g) Cylindrical fibers with hemispherical end and (h) Ellipse inclined at an angle $20^{\circ}$ to Z-axis

RVE is effective. In case of fiber inclusions they may be aligned in one direction or the fibers can be distributed randomly within the RVE itself. Fig. 5 shows both types of fibers arrangement. Due to the manufacturing flexibility, different shaped fibers became easy to produce. With the careful control of the processing parameters, the spherical fibers can be converted in to elliptical shaped fibers. Fig. 6 shows the randomly transversally distributed elliptical fibers with their axis rotated by an angle $20^{\circ}$ to the Z- axis for different volume fraction of the fiber inclusion.
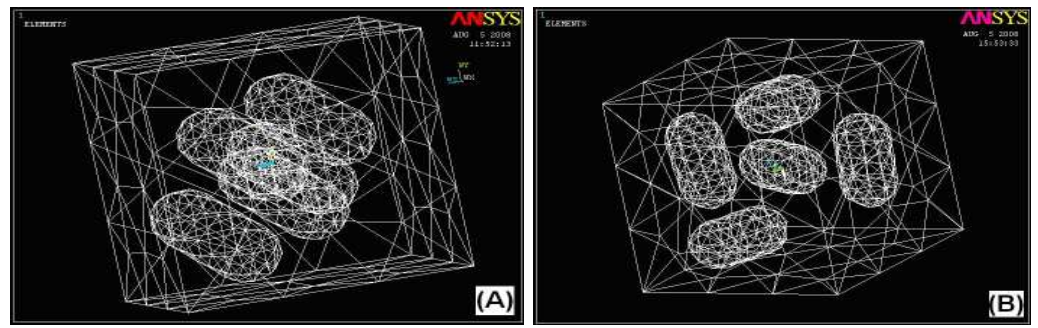

Fig.5. Cylindrical fiber inclusions with hemispherical ends at 10\% volume fraction (a) transversely randomly distributed short fibers (TRDSF), (b) randomly distributed short fibers (RDSF)
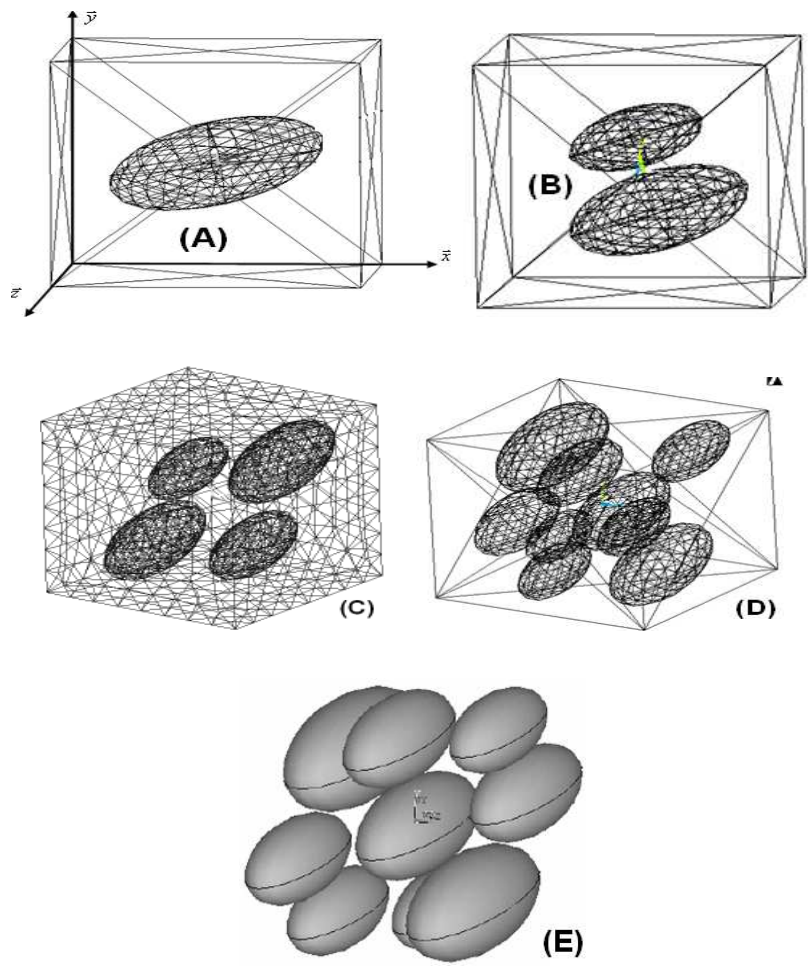

Fig. 6: Transversally randomly distributed elliptical fibers with the axes rotated by $20^{\circ}$ to the Z-axis with the volume fraction of (a) $5 \%$, (b) $10 \%$, (c) $15 \%$, (d) $20 \%$ and (e) $25 \%$ (plotted without RVE). 


\subsection{Numerical homogenization of randomly distributed multi-shaped fiber composites}

The RVE model is the unit cell containing the particle inclusions in the matrix. The elastic constants of the homogenized composite are consisted randomly distributed short multi-shaped non-overlapping fibers. The cubic RVE of volume $\mathrm{L}^{3}$ are obtained through FEA. The modified form of random sequential adsorption algorithm (RSA) is used for a specified distance between neighboring inclusions. For uniformly distributed fiber orientations, and periodicity of the volume elements, the distance between axis of the particle ' $n$ ' and all the cylinders axes $j=1, \ldots,(n-1)$ have to exceed a minimum value $(2 * r+L)$. Where $r$ is the radius of the short multi-shaped fiber and $\mathrm{L}$ is the minimum distance between two adjacent particles, imposed by the practical limitations to create an adequate finite element mesh (Kari et al, 2007) If any surface of the particle ' $i$ ' intersects any of the cubic RVE surfaces, this condition has to be checked with the cylindrical, spherical, cylindrical fibers with hemispherical ends and elliptical inclusions on the opposite surfaces because the microstructure of the composite is periodic [5]. Also the fiber surface should not be very close to the cubic RVE surface as well as corners of the RVE in order to avoid the presence of distorted finite elements during meshing (Berger et al 2005).
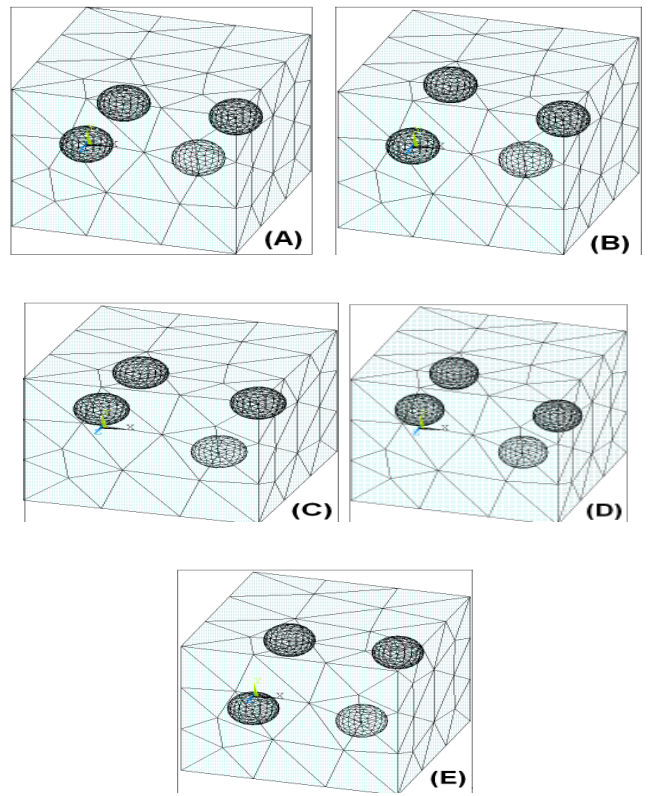

Fig. 7. Four spherical inclusions located at different geometrical locations within the RVE
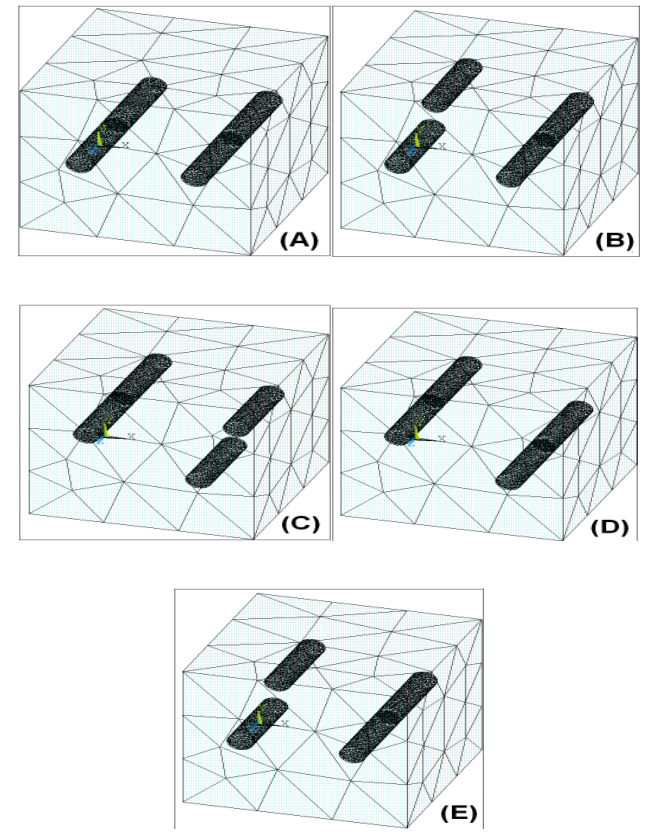

Fig. 8. Four cylindrical inclusions located at different geometrical locations within the RVE 
The RSA algorithm with the combination of the above conditions is used to generate the fiber volumes up to a desired volume fraction of fibers in a composite with uniformly distributed random fiber orientations. Algorithm was used for other shaped fiber inclusions such as spherical, elliptical etc. and combination of above cases, as shown in Figs. 7-8. The volume fraction was varied from $10 \%$ to $60 \%$ for cylindrical fibers, $10 \%$ to $50 \%$ for spherical fibers $5 \%$ to $25 \%$ for elliptical fibers and $3 \%$ to $20 \%$ for combination of spherical, elliptical and cylindrical fibers with each fiber occupying the desired volume fraction. Higher volume fractions and sizes of fibers are suitably adjusted and deposited inside the RVE in descending manner. That is first depositing the largest aspect ratio fibers and after reaching the jamming limit (i.e., no more fibers with that aspect ratio can be deposited), again depositing the next largest possible aspect ratio fibers in the RVE. With this approach the volume fraction achieved is varied keeping in views with the minimum distortion of the finite elements and the adequate mesh. The microscopic structure of the whole composite is calculated by considering a unit cell or representative volume element (RVE). This captures the major features of the underlying microstructure. Finite element calculations were performed with the commercial FE package ANSYS. The matrix and the fibers were meshed with 10 node tetrahedron elements with full integration (Liu et al, 2000)

The ANSYS parametric design language (APDL) was used to calculate the average strains, stresses and effective material properties. The developed APDL scripts in combination with the ANSYS batch processing, provide a powerful tool for the fast calculation of homogenized material properties of composites.

\section{Results and discussion}

\subsection{Influence of volume fraction when RVE remains constant}

Three-dimensional RVE models of fiber inclusions were created containing different shape, size and distribution. For the monodisperse particles, using the described RSA algorithm, the highest volume fraction for the particles were determined. The jamming factor limits the volume fraction of the fiber inclusions in the RVE. The material properties such as elastic modulus (E), Poisson's ratio $(v)$, shear modulus $(\mathrm{G})$ and bulk modulus $(\mathrm{K})$ are considered for the study, which is divided into different categories. The volume fraction of fiber inclusions was varied and the size of the RVE was kept constant. In the subsequent analysis, the volume fraction remains constant and size of the RVE is allowed to change (Keri et al, 2007). The RVE model of each volume fraction is subjected to uni-axial tensile as well as shear deformation along the three axes of co-ordinates. Variation of material properties of cylindrical fibers with hemispherical ends for TRDSF and RDSF loaded composites with change in volume fractions was studied along with change in aspect ratio.
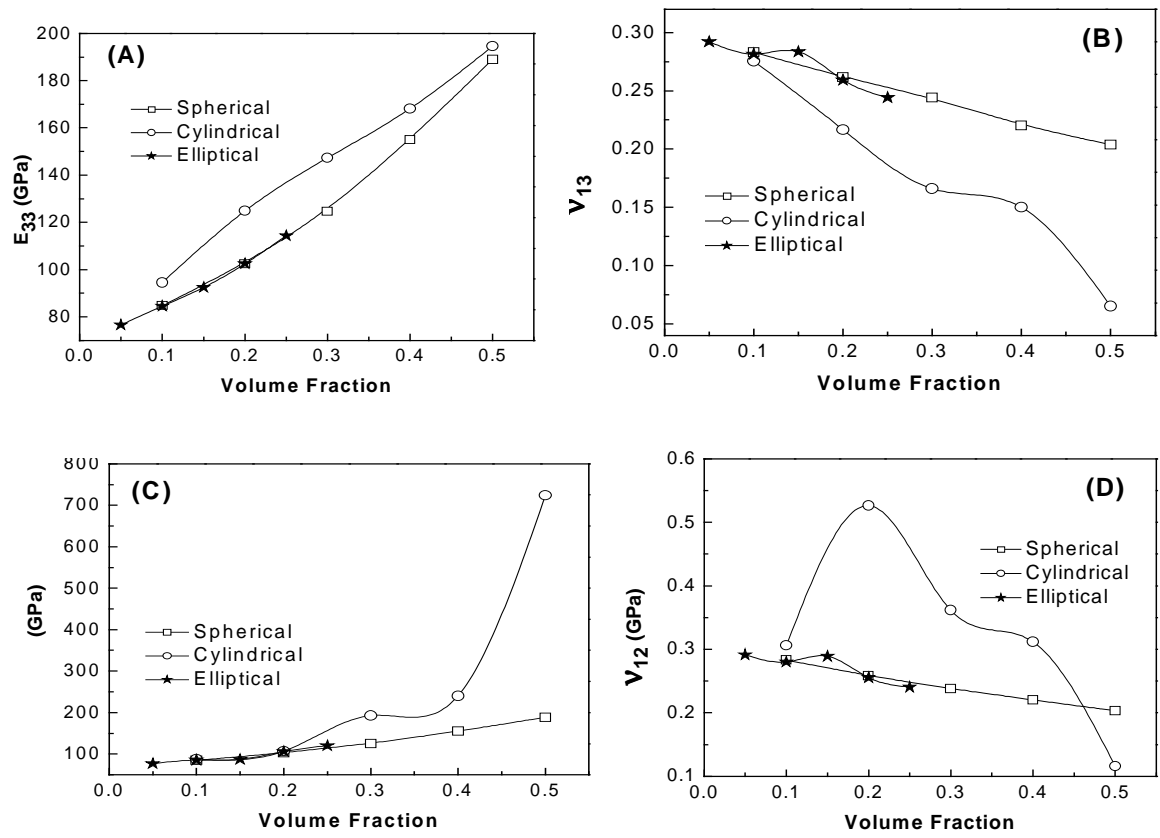

Fig. 9. Variation of effective material properties of the composites for cylindrical, spherical and elliptical fiber inclusion with change of volume fraction (A) longitudinal Young's modulus, (B) longitudinal Poisson's ratios, (C) transverse Young's modulus,

(D) transverse Poisson's ratio 

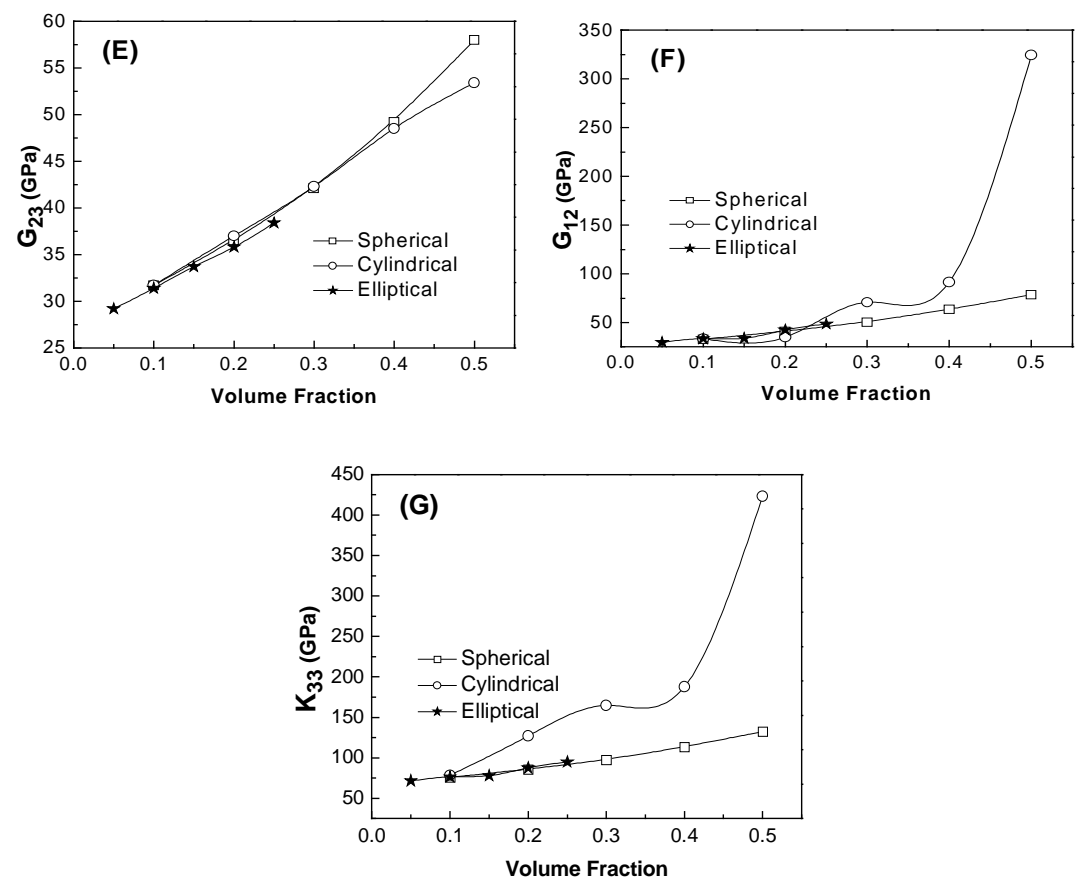

Fig. 9. Variation of effective material properties of the composites for cylindrical, spherical and elliptical fiber inclusion with change of volume fraction (E) longitudinal shear modulus, (F) transverse shear modulus, (G) bulk modulus
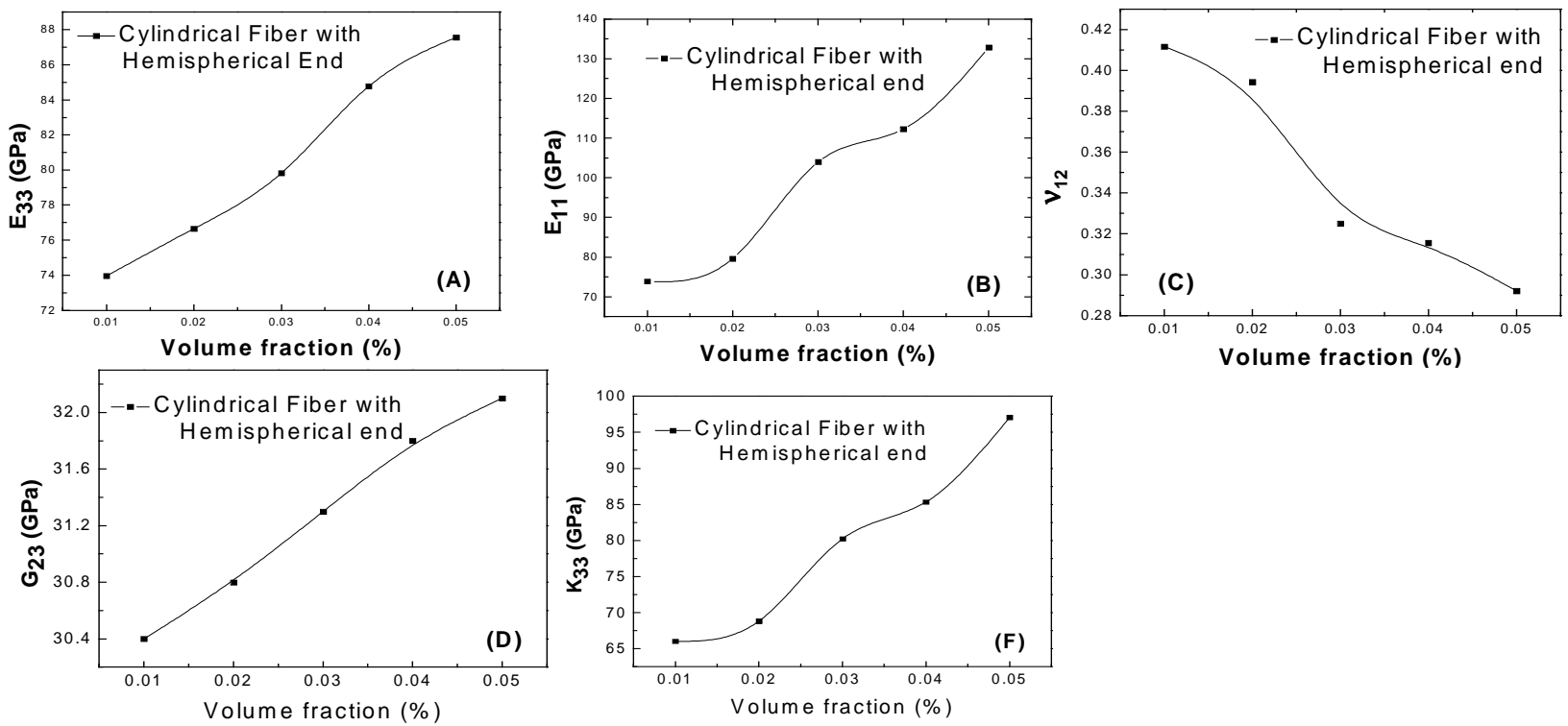

Fig. 10. Variation of effective material properties of cylindrical fiber inclusion with hemispherical ends reinforced composites with change of volume fraction: (A) longitudinal Young's modulus, (B) transverse Young's modulus, (C) transverse Poisson's ratio, (D) longitudinal shear modulus, (E) transverse shear modulus, (F) bulk modulus

Different RVE models with randomly distributed short fibers are considered for each volume fraction, and subjected to uni-axial tensile as well as shear deformation along the three axes of co-ordinates. The ensemble average of the effective material properties at each volume fraction is considered as effective material properties of the total composite at that particular volume fraction. 

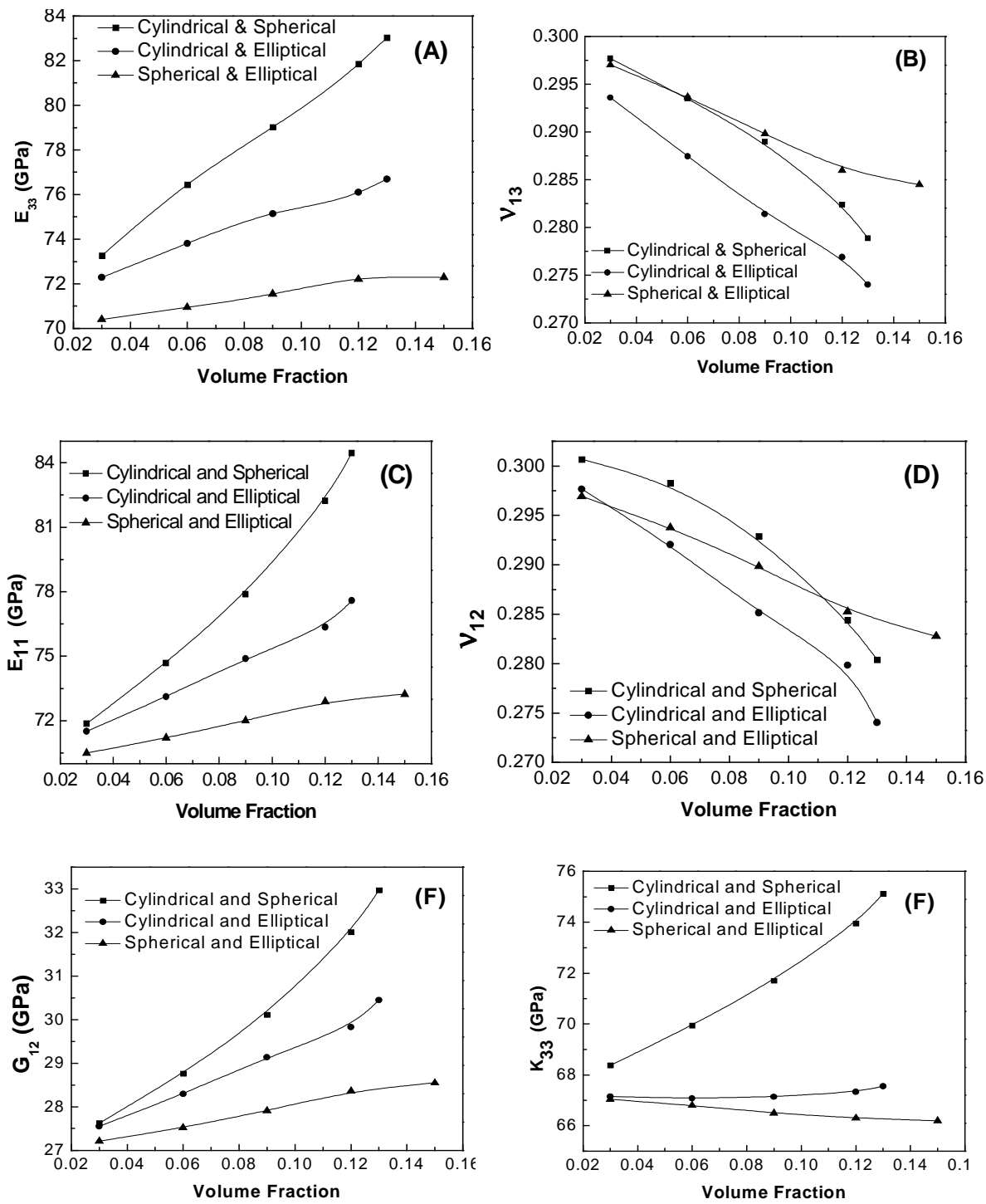

Fig. 11. Variation of effective material properties of the composites for cylindrical \& spherical, cylindrical \& elliptical and spherical \& elliptical fiber inclusion(s) with change of volume fraction (A) longitudinal Young's modulus, (B) longitudinal Poisson's ratios, (C) transverse Young's modulus, (D) transverse Poisson's ratio, (E) transverse shear modulus, (F) bulk modulus
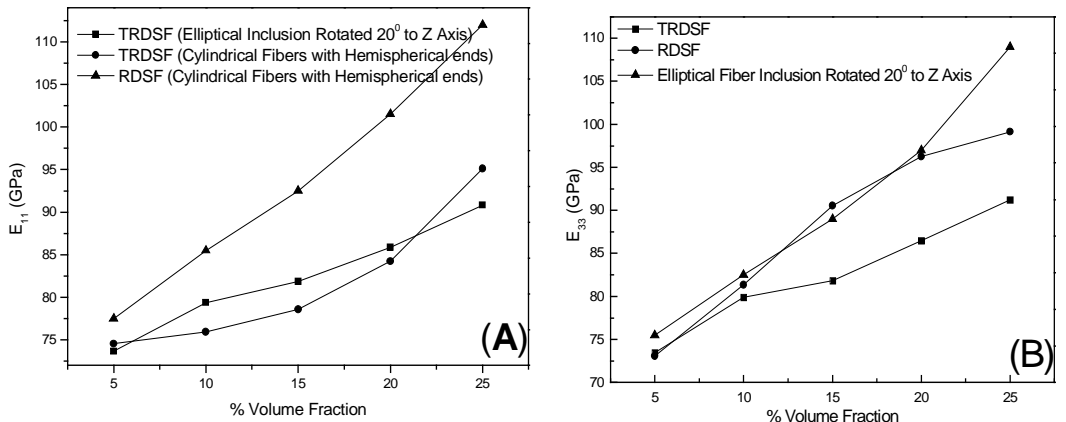

Fig. 12. Variation of effective material properties of the composites for transversely randomly distributed elliptical inclusion rotated at $20^{\circ}$ to the Z-axis, transversely randomly distributed cylindrical fibers with hemispherical ends and randomly distributed cylindrical fibers with hemispherical ends and comparison with different analytical results (A) transverse Young's modulus, (B) longitudinal Young's modulus 

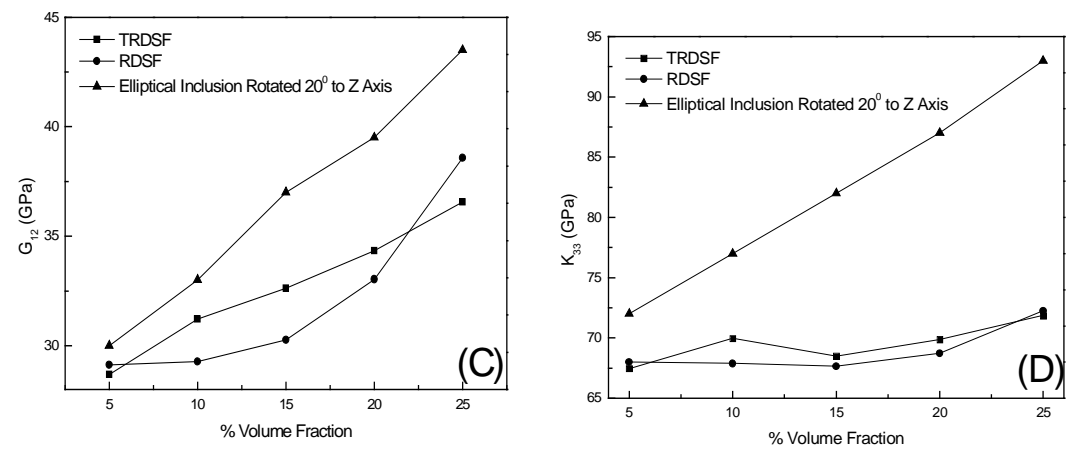

Fig. 12. Variation of effective material properties of the composites for transversely randomly distributed elliptical inclusion rotated at $20^{\circ}$ to the Z-axis, transversely randomly distributed cylindrical fibers with hemispherical ends and randomly distributed cylindrical fibers with hemispherical ends and comparison with different analytical results $(C)$ transverse shear modulus,

(D) bulk modulus

Figures (9-12) show the variation of effective material properties such as Young's modulus, Poisson's ratio, shear modulus, and bulk modulus respectively, with the change in volume fraction of fiber inclusion. Fig. 9(A) shows the variation of modulus in the fiber direction, $\mathrm{E}_{33}$, with the variation of the volume fraction of fiber inclusion. This shows the increase of $\mathrm{E}_{33}$ with increase of volume fraction for single particle inclusion (i.e. cylindrical, spherical, elliptical fibers). It is observed that the highest numerical value of the result corresponds to the larger volume fraction. This may be due to the fact that when the resin content of fiber is more, then flaws appeared due to micro cracks and voids. This may be present in the matrix that acts as the point of stress concentration and fractured at lower load (Keri et al, 2007). With the increase of volume fraction of fiber content the above defects minimizes and the proper interfacial bonding is achieved. After certain limit of the quantity of the resin content, it's difficult to simulate the results due to the jamming effect. In all the analyses the RVE models containing the cylindrical fiber inclusion give maximum value. The similar trend is also observed for $\mathrm{E}_{11}, \mathrm{G}_{12}, \mathrm{G}_{33}$ and $\mathrm{K}_{33}$ in all models. The results for the spherical and elliptical inclusions are overlapped, due to comparable surface geometry. The values of the Poisson's ratio are decreased with the increasing of volume fraction of the fiber inclusion. The uneven distribution of Poisson's ratio for $v_{12}$ and $v_{13}$ are not clear.

Also, rounded sphere-cylinders with the combination of cylindrical body and two hemispheric caps at the both ends removing the singularities are considered, as shown in Fig. 10. It is clear that fibers do not have rounded caps but it can be considered as a minor change in shape compared to plane cylindrical approach (Ramos et al, 2001). The material properties are calculated corresponding to the different volume fraction of fiber content. The Young's modulus, shear modulus and bulk modulus is found to be increases with the increase of volume fraction. But the values are found to be lower than the earlier cases for cylindrical inclusion alone. This might be due to the fact of lower interfacial bonding in the smooth surfaces. So when load is applied to this type material, it offers less resistance. Finally, consider the pair inclusions of cylindrical- spherical, spherical-elliptical, ellipticalcylindrical and pairs of particle inside the RVE, as shown in Fig. 11. Due to jamming effect, the volume fraction of fiber inclusion varies in different models. The cylindrical-spherical combination gives the better results than the other two combinations. Fig. 12 shows the variation of effective materials properties with the ratio of major to minor axis (ellipse rotated through $20^{\circ}$ along Z-axis with 5\% volume fraction) and rotation of major axis along Z-direction. Material properties versus volume fraction of TRDSF elliptical inclusion $\left(\mathrm{a} / \mathrm{b}=2\right.$, angle of rotation $=20^{\circ}$ to Z-axis) is represented along with the TRDSF and RDSF cylindrical fibers with the hemispherical end.

\subsection{Influence of size of RVE on the effective material properties \& volume fraction of $30 \%$}

The heterogeneous materials are analyzed through the RVE technique having volume, which is sufficiently large enough for the statistical representation of composites. It is considered to be capable of sampling of all micro-structural heterogeneity that occurs in the composite (Wang, 1998). Such type of RVE is capable of reproducing the bulk properties of the composites. There is a minimum size for the RVE (with short fibers), which is required to give appropriate effective material properties of a macroscopic composite structure. If the size of the RVE considered is less than the minimum size required, it may lead to a wrong prediction of effective material properties. Numerical homogenization techniques can help in determining the critical size of the RVE. In order to determine the minimum size of RVE, identical short fiber particles were considered and by changing the size of the cubic RVE, the effective material properties were obtained for 30\% volume fraction. Figs. 13 and 14 show the variation of effective material properties in response to the change in size of the RVE. With identical aspect ratio of fibers (length of fiber/diameter of fiber) using this algorithm, it is possible to generate up to $30 \%$ volume fractions RVE models. But, it is not possible to generate higher volume fraction RVE models because of the jamming limit. Studies are presented to determine the effect of the size of RVE on the effective material properties of these composites. 
For this purpose, we considered the cubic length of the RVE that varies from 0.4 to 2 unit length. The single inclusion like cylindrical, spherical and elliptical is considered. The cylindrical inclusion RVE model gives the best results in comparison to the other cases. In the same analysis also assumed that the combine cases of elliptical-cylindrical, cylindrical-spherical and sphericalelliptical fiber inclusion. The spherical-cylindrical combination gives the best result in comparison to the other two varieties as mentioned earlier.
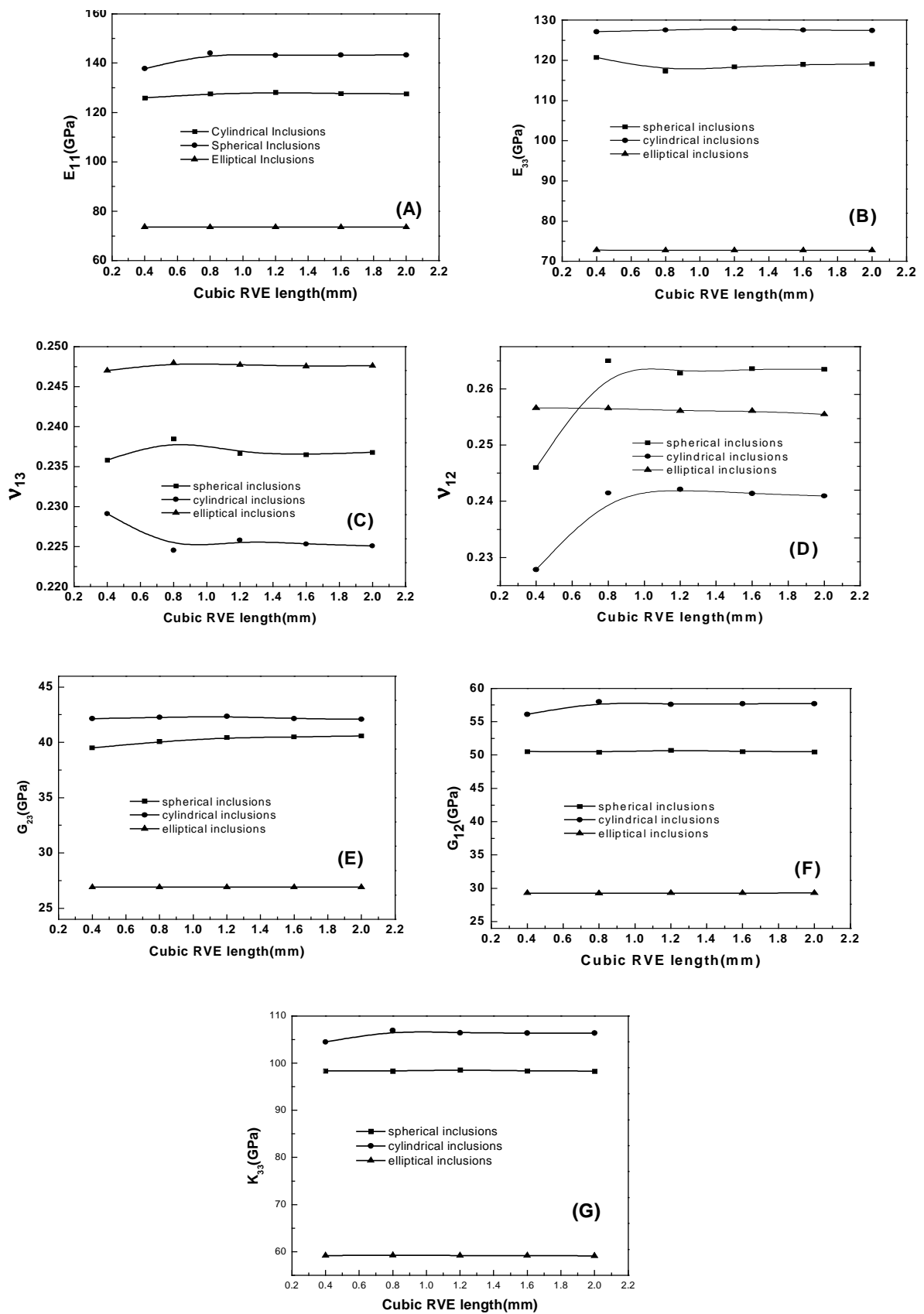

Fig. 13. Variation of effective material properties of the composites for cylindrical, spherical and elliptical fiber inclusion(s) with change in cubic RVE length (A) Transverse Young's modulus, (B) longitudinal Young's modulus, (C) longitudinal Poisson's ratios, (D) transverse Poisson's ratio, (E) longitudinal shear modulus, (F) transverse shear modulus, (G) bulk modulus 

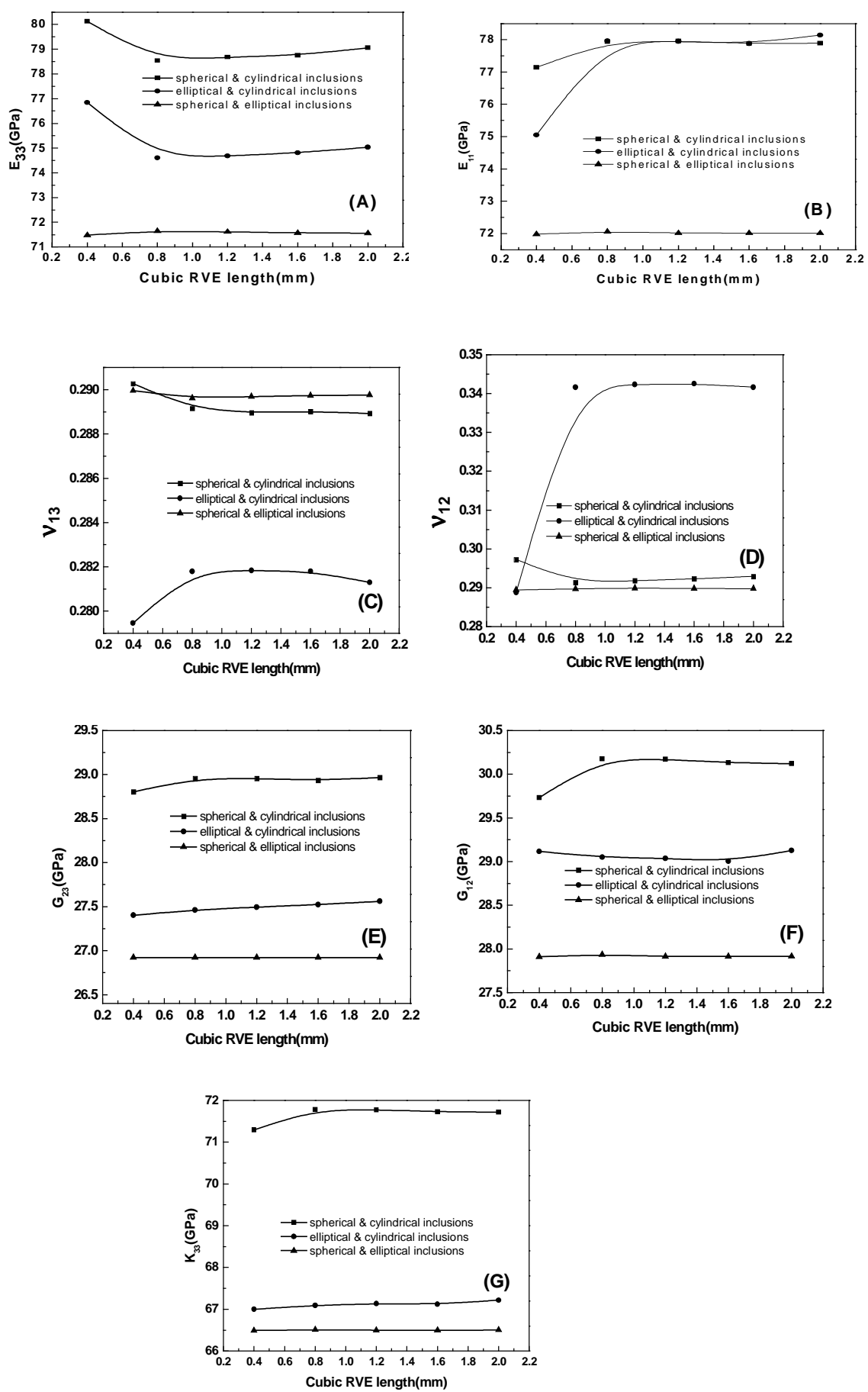

Fig. 14. Variation of effective material properties of the composites for cylindrical- spherical, cylindrical - elliptical and spherical \& elliptical fiber inclusion(s) with change in cubic RVE length (A) longitudinal Young's modulus, (B) transverse Young's modulus, (C) longitudinal poisson's ratios, (D) transverse poisson's ratio, (E) longitudinal shear modulus, (F) transverse shear modulus, $(\mathrm{G})$ bulk modulus

\subsection{Influence of the position of fiber inclusions and number of inclusions in RVE-volume fraction $2.5 \%$}

The influence of position of fiber inclusion is an important aspect, which is studied along with the distribution of fibers at different locations within the RVE. First, considered a spherical fiber inclusion, as shown in Fig.7 and change its position by varying its centre coordinates within the RVE. The coordinate points for the geometrical centre of the sphere(s) are shown in the Table-2. In this analysis the volume fraction of fiber remains constant where as only the number of sphere and its position changes. 
Table 2. Coordinate points of the spherical inclusion(s) for different location points within the RVE

\begin{tabular}{|l|l|l|l|l|l|}
\hline 4 spheres & $\begin{array}{c}\text { Coordinates } \\
\text { for the } \\
\text { position 1 }\end{array}$ & $\begin{array}{c}\text { Coordinates } \\
\text { for the } \\
\text { position 2 }\end{array}$ & $\begin{array}{c}\text { Coordinates } \\
\text { for the } \\
\text { position 3 }\end{array}$ & $\begin{array}{c}\text { Coordinates } \\
\text { for the } \\
\text { position 4 }\end{array}$ & $\begin{array}{c}\text { Coordinates } \\
\text { for the } \\
\text { position 5 }\end{array}$ \\
\hline $\mathrm{X} 1$ & 0.5 & 0.5 & 0.5 & 0.5 & 0.5 \\
\hline $\mathrm{Y} 1$ & 1 & 1.3 & 1.3 & 1.3 & 1.3 \\
\hline $\mathrm{Z} 1$ & 0.5 & 0.5 & 0.5 & 0.5 & 0.5 \\
\hline $\mathrm{X} 2$ & 0.5 & 0.5 & 0.5 & 0.5 & 0.5 \\
\hline $\mathrm{Y} 2$ & 1 & 1 & 1.3 & 1.3 & 0.8 \\
\hline $\mathrm{Z} 2$ & 1.5 & 1.5 & 1.5 & 1.5 & 1.5 \\
\hline $\mathrm{X} 3$ & 1.5 & 1.5 & 1.5 & 1.5 & 1.5 \\
\hline $\mathrm{Y} 3$ & 1 & 1 & 1 & 0.8 & 1.3 \\
\hline $\mathrm{Z} 3$ & 0.5 & 0.5 & 0.5 & 0.5 & 0.5 \\
\hline $\mathrm{X} 4$ & 1.5 & 1.5 & 1.5 & 1.5 & 1.5 \\
\hline $\mathrm{Y} 4$ & 1 & 1 & 0.8 & 0.8 & 0.8 \\
\hline $\mathrm{Z} 4$ & 1.5 & 1.5 & 1.5 & 1.5 & 1.5 \\
\hline
\end{tabular}

Table 3. Coordinate points of the cylindrical inclusion(s) for different location points within the RVE

\begin{tabular}{|c|c|c|c|c|c|}
\hline 4 cylinders & $\begin{array}{c}\text { Coordinates for } \\
\text { the position 1 }\end{array}$ & $\begin{array}{c}\text { Coordinates } \\
\text { for the } \\
\text { position 2 }\end{array}$ & $\begin{array}{c}\text { Coordinates } \\
\text { for the } \\
\text { position 3 }\end{array}$ & $\begin{array}{c}\text { Coordinates } \\
\text { for the } \\
\text { position 4 }\end{array}$ & $\begin{array}{c}\text { Coordinates } \\
\text { for the } \\
\text { position 5 }\end{array}$ \\
\hline $\mathrm{X} 1$ & 0.5 & 0.5 & 0.5 & 0.5 & 0.5 \\
\hline $\mathrm{Y} 1$ & 1 & 1.3 & 1.3 & 1.3 & 1.3 \\
\hline $\mathrm{Z} 1$ & 0.5 & 0.5 & 0.5 & 0.5 & 0.5 \\
\hline $\mathrm{X} 2$ & 0.5 & 0.5 & 0.5 & 0.5 & 0.5 \\
\hline $\mathrm{Y} 2$ & 1 & 1 & 1.3 & 1.3 & 0.8 \\
\hline $\mathrm{Z} 2$ & 1.5 & 1.5 & 1.5 & 1.5 & 1.5 \\
\hline $\mathrm{X} 3$ & 1.5 & 1.5 & 1.5 & 1.5 & 1.5 \\
\hline $\mathrm{Y} 3$ & 1 & 1 & 1 & 0.8 & 1.3 \\
\hline $\mathrm{Z} 3$ & 0.5 & 0.5 & 0.5 & 0.5 & 0.5 \\
\hline $\mathrm{X} 4$ & 1.5 & 1.5 & 1.5 & 1.5 & 1.5 \\
\hline $\mathrm{Y} 4$ & 1 & 1 & 0.8 & 0.8 & 0.8 \\
\hline $\mathrm{Z} 4$ & 1.5 & 1.5 & 1.5 & 1.5 & 1.5 \\
\hline
\end{tabular}

The positions of these inclusions were changed within the RVE, as given in Table-3 and the comparison of effective material properties are represented in Figs. 15 and 16. The result shows that the single spherical/cylindrical fiber inclusion gives better material properties.

The spherical inclusion suffers from the drawback that a large volume fraction has to be used to achieve the strength as compared to elliptical and multi shaped inclusion composites. The composites with cylindrical inclusions can be used where very high strength material is required. Also, it was found that the change of position as well as numbers does not alter the properties of the RVE.
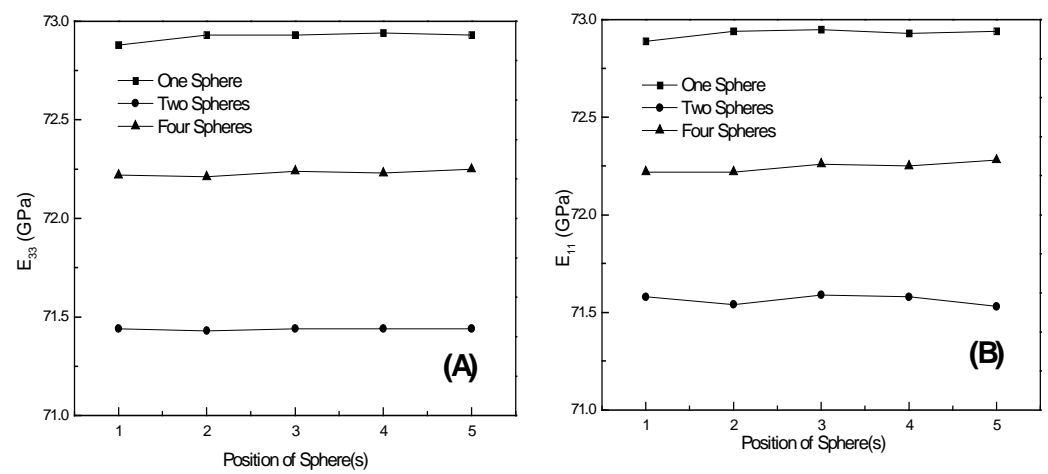

Fig. 15. Variation of effective material properties of the composites for one sphere, two spheres and four spheres inclusion(s) with change in absolute position at different locations (A) longitudinal Young's modulus, (B) transverse Young's modulus 

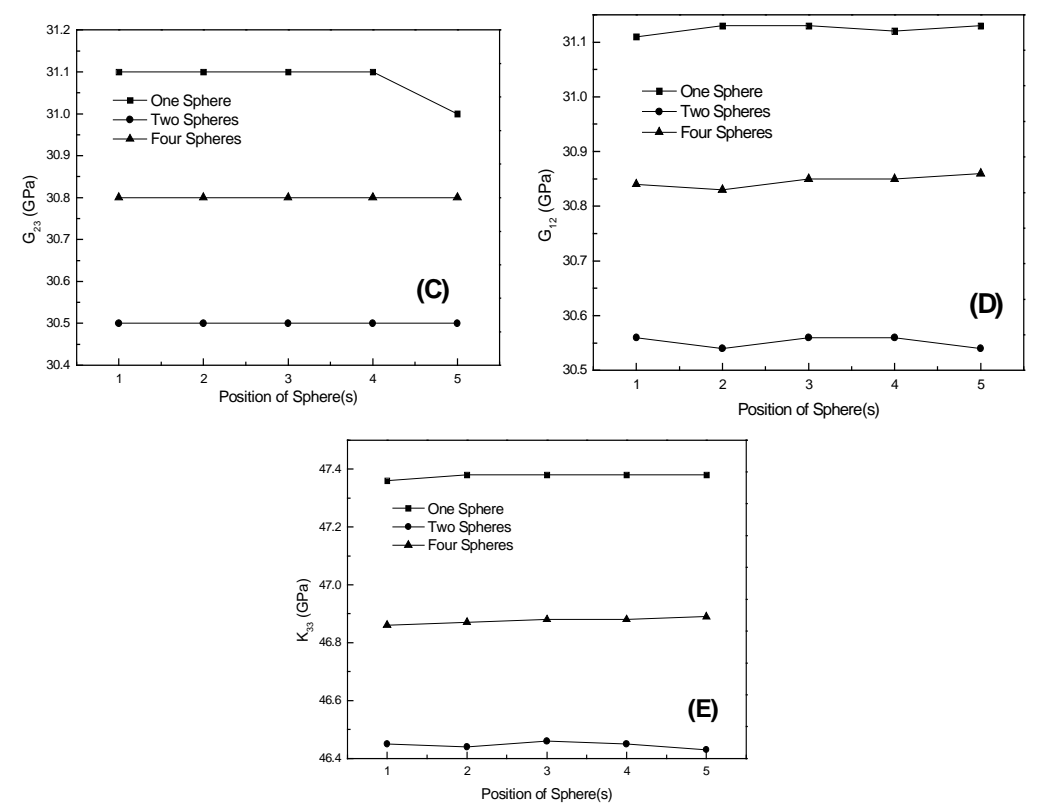

Fig. 15. Variation of effective material properties of the composites for one sphere, two spheres and four spheres inclusion(s) with change in absolute position at different locations (C) longitudinal shear modulus, (D) transverse shear modulus, (E) bulk modulus
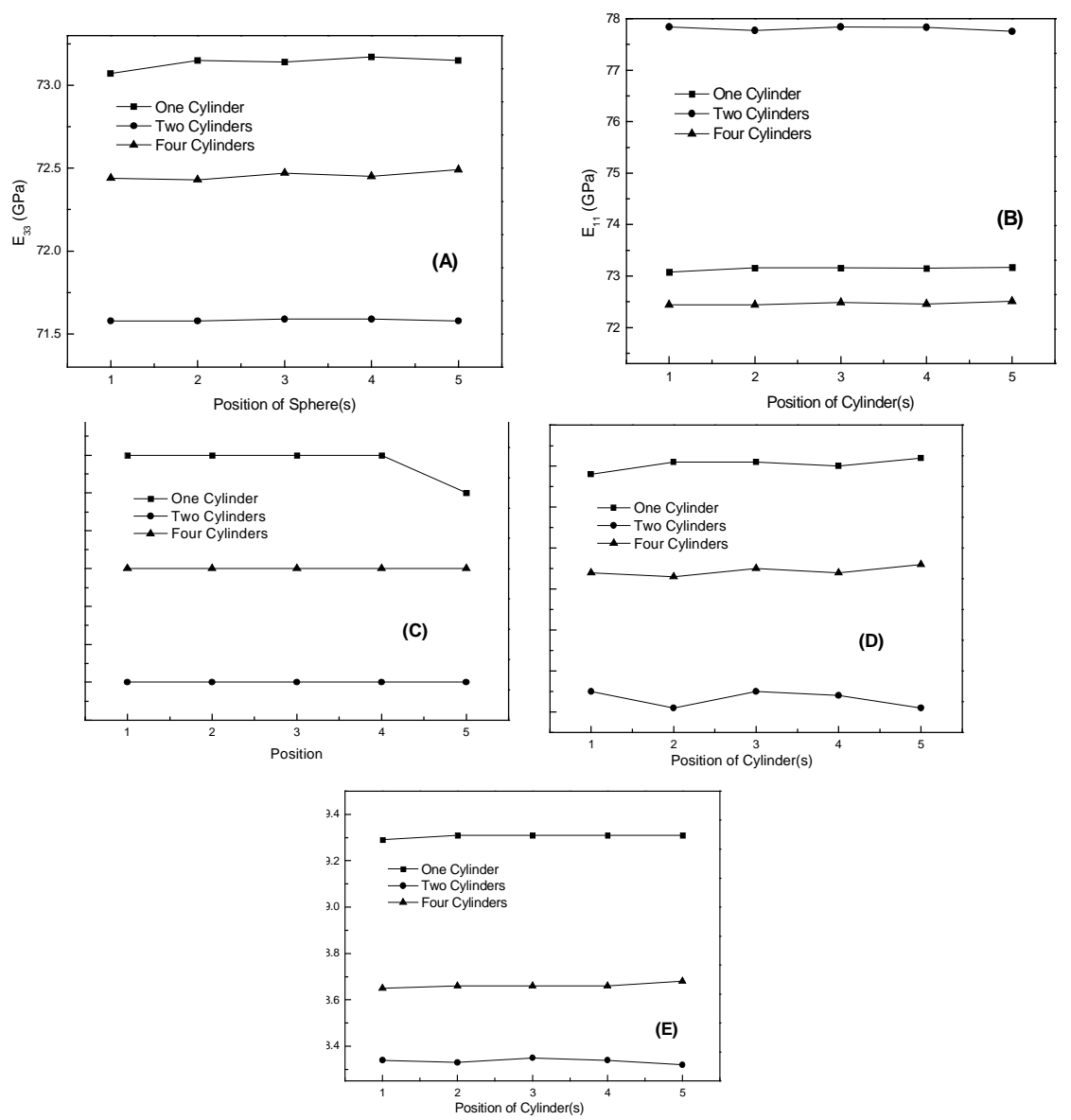

Fig. 16. Variation of effective material properties of the composites for one cylinder, two cylinders and four cylinders inclusion(s) with change in absolute position at different locations (A) longitudinal Young's modulus, (B) transverse Young's modulus, (C) longitudinal shear modulus, (D) transverse shear modulus, (E) bulk modulus 


\subsection{Influence of volume fraction on the cylindrical fiber with hemispherical ends}

The effect of the material properties with the change of the volume fraction of the randomly distributed (RDCFH), transversely randomly distributed cylindrical fibers with the hemispherical ends (TRDCFH) and transversely randomly distributed elliptical fiber rotated at an angle $20^{\circ}$ to the Z-axis $\left(\mathrm{TRDSF} / 20_{\mathrm{z}}{ }^{\circ}\right)$ was considered for the analysis. It was observed that, the numerical value of the transverse Young's modulus $\left(\mathrm{E}_{11}\right)$ at $5 \%$ volume fraction of fiber inclusion is almost the same for the TRDSF and RDSF arrangement of cylindrical fibers with hemispherical ends but there is an improvement of $3 \%$ in the case of elliptical fiber inclusion (Liu et al 2000). Corresponding to 10, 15 and 20\% volume fraction, the randomly distributed cylindrical fibers with hemispherical ends shows 4.5, 4.1 and $1.94 \%$ improvements over the transversely randomly distributed similar kind of fibers. At higher volume fraction i.e. $25 \%$, the nature of the TRDSF for the hemispherical ended cylindrical fiber get reversed and an improvement of $4.7 \%$ is observed. The TRDSF $/ 20_{\mathrm{z}}{ }^{2}$ shows an improvement of 4, 13, 17.7, 20.5 and $17.7 \%$ on TRDCFH for 5, $10,15,20$ and $25 \%$ volume fraction. There is an increase in trend is observed for $\mathrm{E}_{33}$ with the variation of volume fraction for $\mathrm{RDCFH}, \mathrm{TRDCFH}$ and TRDSF $/ 2 \mathrm{O}_{\mathrm{z}}{ }^{\circ}$. At lower volume fraction, say at $5 \%$ and $10 \%$, there is no considerable amount of improvements in the properties is observed for $\mathrm{E}_{33}$ but at higher volume fraction, say at $25 \%$, the TRDSF/20 ${ }_{\mathrm{z}}{ }^{0}$ shows $9 \%$ and $16.3 \%$ improvement over RDCFH and TRDCFH. Similar trend is observed for $\mathrm{G}_{12}$ where the TRDSF/20 ${ }_{\mathrm{z}}^{0}$ shows superior properties than RDCFH and TRDCFH over the increase in the volume fraction of the fiber inclusion. For $\mathrm{K}_{33}$, the curve for the RDCFH and TRDCFH almost overlap with each other but there is a linear increase in the properties is observed for TRDSF/20 ${ }_{\mathrm{z}}{ }^{\circ}$. TRDSF $/ 20_{\mathrm{z}}{ }^{0}$ produces $5.5 \%$ improvement for $5 \%$ volume fraction and $22.5 \%$ improvements at $25 \%$ volume fraction over RDCFH and TRDCFH.

\subsection{Variation of material properties}

The effect of aspect ratio is an important consideration for the analysis of composite performance. If changes the aspect ratio from the numerical value 1 to 6 . The property like $E_{33}, E_{11}$ and $G_{12}$ remains almost linear for the different values of aspect ratios for TRDCFH, as can be identified from Fig. 17. The curves for the RDCFH show some irregular deviation and the cause is unknown at this moment. Also, from the earlier analysis it was observed that TRDSF/ $20_{z}{ }^{0}$ shows better properties over the range of the volume fraction of the fiber inclusion.
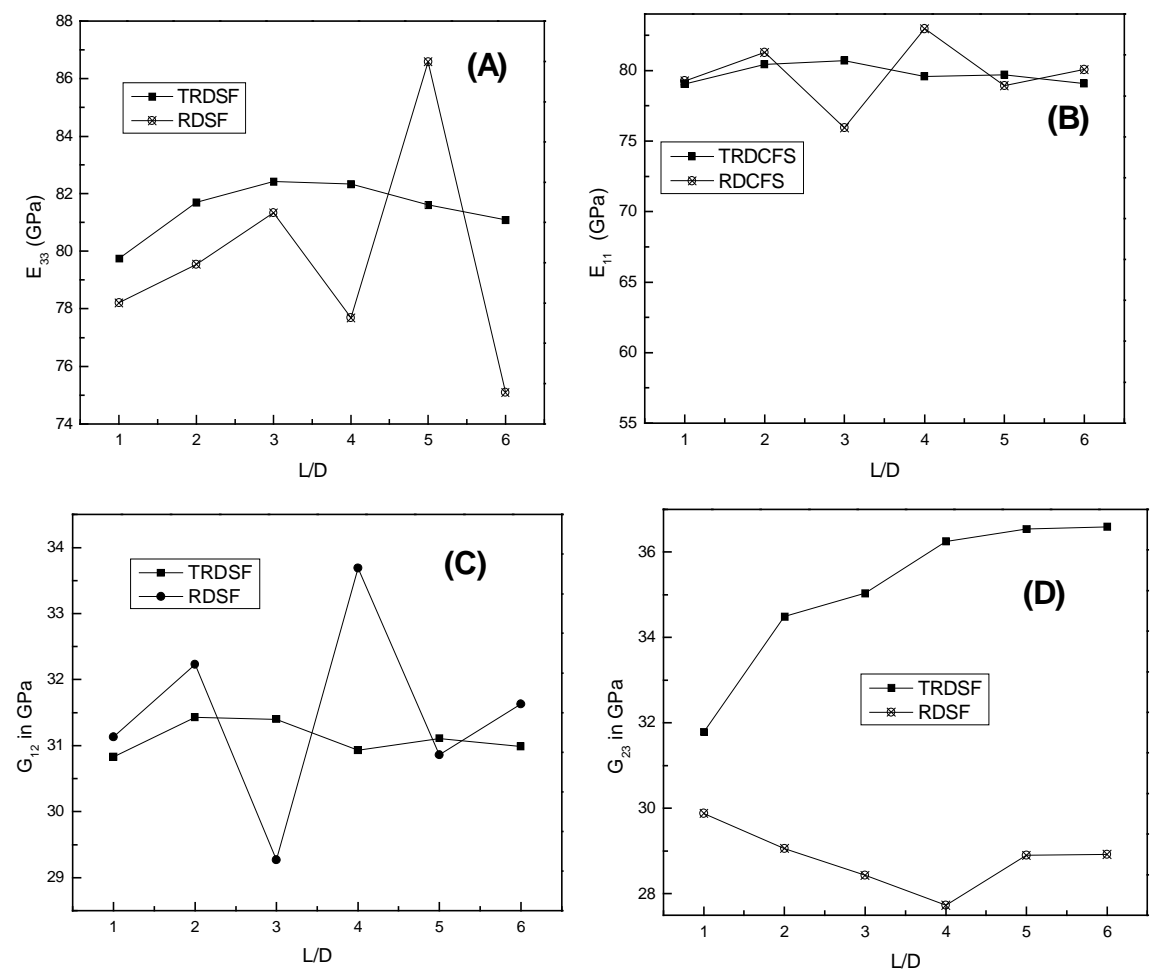

Fig. 17. Variation of effective material properties of the composites for cylindrical fiber with hemi spherical end inclusion(s) with the variation of aspect ratio and comparison with different analytical results (A) longitudinal Young's modulus, (B) transverse Young's modulus, (C) transverse shear modulus, (D) longitudinal shear modulus 


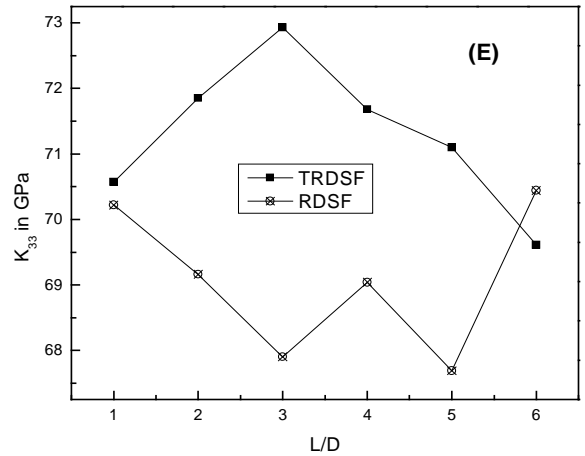

Fig. 17. Variation of effective material properties of the composites for cylindrical fiber with hemi spherical end inclusion(s) with the variation of aspect ratio and comparison with different analytical results (E) bulk modulus

Fig. 18(A) shows that, the material properties do not change with the changes of aspect ratio for TRDSF/20 ${ }_{\mathrm{z}}{ }^{0}$. The effect of the angular positioning of the elliptical fiber in the RVE is an important aspect and need complete analysis. For this purpose, the volume fraction of the elliptical fiber inclusion is kept constant i.e. 5\% and the ratio of the major axis to the minor axis is 2, as can be seen from Fig. 18 (B). It was found that, the various properties such as $E_{33}, E_{11}, K_{13}, G_{13}$ and $G_{12}$ are unaffected by the different angular positions of elliptical fiber i.e. $0^{0}$ to $25^{0}$ with $5^{0}$ increments. There is an unexpected drop is observed for all the above properties at the $10^{\circ}$ angular position.
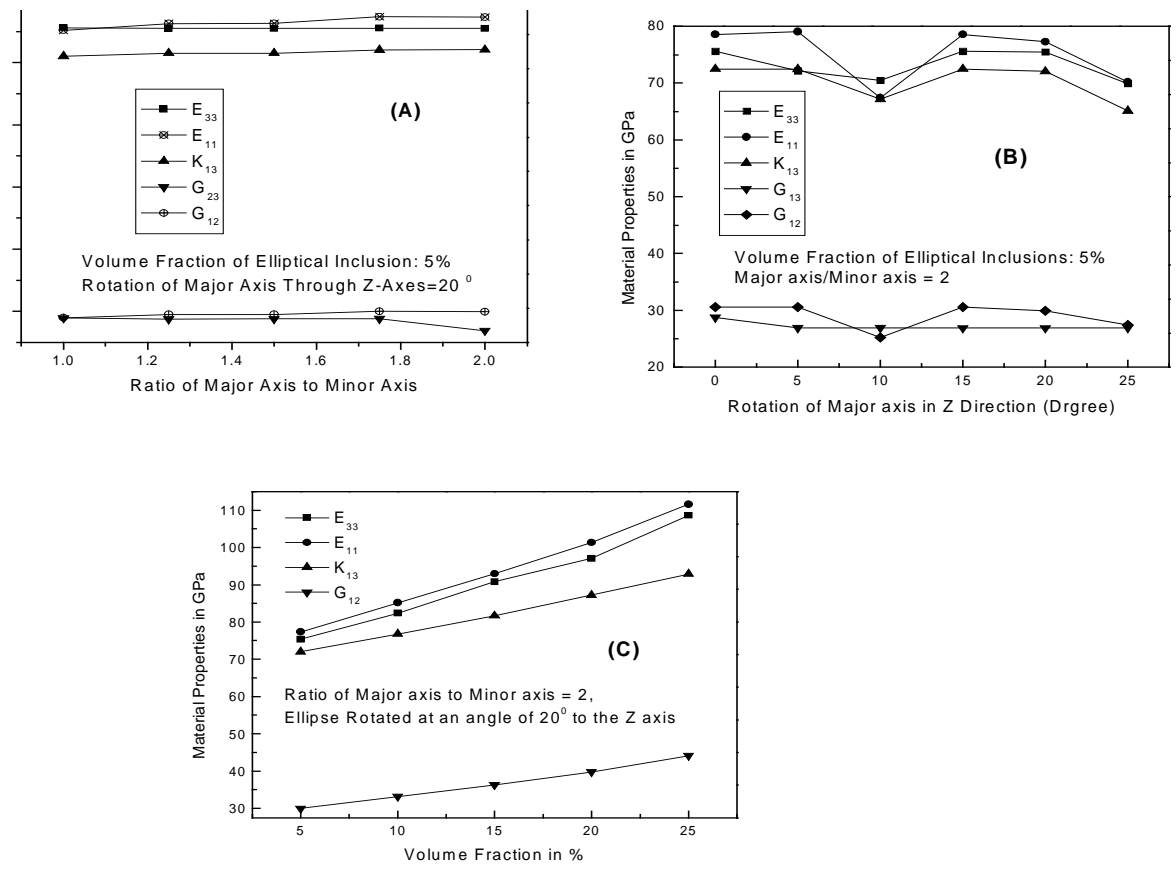

Fig. 18. Variation of effective material properties of the composites for the elliptical fiber inclusion (A) ratio of major axis to minor axis of the ellipse $=2$ and rotated at an angle $20^{\circ}$ to the Z-axis with the variation of the ratio of major axis to minor axis, (B) at constant volume fraction of $5 \%$ with the ratio of the major axis to the minor axis $=2$ with the variation of the angle of rotation of the major axis along the $\mathrm{Z}$ direction, (C) at a particular angular position 200 to the $\mathrm{Z}$ axis and constant value of the ratio of major axis to minor axis $=2$ with the variation of the volume fraction.

The elliptical fiber with the ratio of Major axis to the Minor axis 2, which is placed in the RVE at a constant angular position $20^{\circ}$ to the Z-axis is consider for the analysis, as shown in Fig. 18(C). The volume fraction of the fiber inclusion was varied from 5\% to $25 \%$ with increments of $5 \%$. It was found that the properties like $\mathrm{E}_{33}, \mathrm{E}_{11}, \mathrm{~K}_{12}$ and $\mathrm{G}_{12}$ gradually increases with the increase of the volume fraction of the fiber inclusion (Berger et al, 2006). The increments in the properties for $\mathrm{E}_{33}, \mathrm{E}_{11}, \mathrm{~K}_{12}$ and $\mathrm{G}_{12}$ from $5 \%$ to $25 \%$ volume fraction are $30.65,30.67,22.45$ and $32 \%$ respectively. 


\section{Conclusions}

Numerical homogenization tools have been developed for the evaluation of the effective material properties of the short fiber composites. The results showed that the effective material properties depend mainly on the volume fraction. Also, Young's modulus, shear modulus and bulk modulus properties can be improved with the increase of fiber volume fraction. Volume fraction for cylindrical, spherical and elliptical fiber inclusions can not be increased more than $50 \%$, where as in the combined cases, it should be in between 3 to $15 \%$ to avoid the jamming effect. This statement is valid for linear elastic case for the evaluation of effective material properties only.

\section{Acknowledgement}

The authors will like to acknowledge DST, New Delhi, India and DAAD, Germany for their financial support.

\section{References}

Agbossou A., Pastor J. 1997. Thermal Stresses and Thermal Expansion Coefficients of n-Layered Fiber-Reinforced Composites, Composite Science \& Technology, Vol57, pp. 249-260.

Berger H., Kari S., Gabbert U., Rodriguez-Ramos R., Guinovart-Diaz R., Bravo-Castillero J. 2005. An analytical and numerical approach for calculating effective material coefficients of piezoelectric fiber composites, International Journal of Solid Structure, Vol. 21-22, pp 5692-5714.

Berger H., Kari S., Gabbert U., Ramos Rodriguez R., Guinovart-Diaz R., Otero J.A., Bravo- Castillero J. 2006. Unit cell models of piezoelectric fiber composites for numerical and analytical calculation of effective properties, Smart Material Structure, Vol. 15 , pp. 451-458.

Hashin Z. 2002. Thin interphase/imperfect interface in elasticity with application to coated fiber composites, Journal of Mech Phys Solids, Vol. 50, pp. 2509-2537.

Kari S., Berger H., Rodriguez-Ramos R., Gabbert U. 2007. Computational evaluation of effective material properties of composites reinforced by randomly distributed spherical particles, Computer Structures, Vol. 77, pp. 223-231.

Kari S. 2006. Micro-mechanical Modeling and numerical homogenization of fiber and particle reinforced composites, Reihe 18, Mechanik, Nr 309, Ph.D. thesis, VDI Verlag GmbH, Germany.

Kari S., Berger H., Gabbert U. 2007. Numerical evaluation of effective material properties of randomly distributed short cylindrical fiber composites, Computational Material Science, Vol. 39, pp. 198-204.

Liu Y..J., Xu N., Luo J.F. 2000. Modeling of interphases in fiber-reinforced composites under transverse loading using the boundary element method, J Appl Mech, Vol. 67, pp. 41-49.

Ramos R.R., Sabina F.J., Diaz R.G., Castillero J.B. 2001. Closed- form expressions for the effective coefficients of fiberreinforced composite with transversely isotropic constituents. I: Elastic and square symmetry, Mech Mater, Vol. 33, pp. 223235.

Wang J.S. 1998. Random sequential adsorption, series expansion and Monte Carlo simulation, Physica-A, Vol. 254, pp. 179-184.

\section{Biographical notes}

Dr. V.K. Srivastava received M.Tech and Ph.D. degree from Institute of Technology, BHU, Varanasi in 1979 and 1987, respectively. He is a Professor in the Department of Mechanical Engineering, Institute of Technology, BHU, Varanasi, India. He is Fellow of IE, India. His research interests include composite materials, ceramic composites. He has published more than 130 papers in referred international journals and conferences. He is editorial member of various International journals.

Dr. Ulrich Gabbert is a senior Professor and Head of Institute of Mechanics, Otto-von-Guericke University of Magdeburg, Magdeburg, Germany.

Dr. H. Berger is a Professor Assistant, Institute of Mechanics, Otto-von-Guericke University of Magdeburg, Magdeburg, Germany.

Miss Shraddha Singh is Ph.D. student, School of Materials Science \& Technology, Institute of Technology, BHU, Varanasi, India.

Received February 2011

Accepted February 2011

Final acceptance in revised form March 2011 